\title{
Caustic ingestion in children: A 6-year retrospective study
}

\author{
Cristina Lovera, ${ }^{1}$ Emanuele Castagno, ${ }^{2}$ Claudia Bondone, ${ }^{2}$ Fulvio Ricceri, ${ }^{3,4}$ Antonio F. Urbino ${ }^{2}$ \\ ${ }^{1}$ Division of Pediatrics, A.O. Santa Croce e Carle, Cuneo; ${ }^{2}$ A.O.U. Città della Salute e della Scienza di \\ Torino - Regina Margherita Children's Hospital, Department of Pediatric Emergency, Turin; ${ }^{3}$ Department \\ of Clinical and Biological Sciences, University of Turin, Turin; ${ }^{4}$ Unit of Epidemiology, Regional Health \\ Service, ASL TO3, Grugliasco (TO), Italy
}

\begin{abstract}
Some aspects of the management of caustic ingestion in children are still debated. We aimed to determine the predictive value of epidemiological and clinical features of caustic ingestion, and to define guidelines adherence. This is a retrospective study on children $\leq 18$ years referred to our Emergency Department over 6 years because of caustic ingestion. Statistical significance at $\mathrm{p}<0.05$. Overall, 107 caustic ingestions were identified, mainly accidental $<5$ years $(87.85 \%)$. Bleach was the most common caustic (46.73\%). Forty-six patients underwent endoscopy; $57.38 \%$ children who should have endoscopy on guidelines, actually did not ( $p<0.0001)$, but no complications were reported. No or minimal lesions were reported in asymptomatic children; $\geq 2$ symptoms correlated with the severity of lesions $(\mathrm{p}<0.0002)$. Therapy of severe cases was correct; overtreatment was observed for minor injuries $(\mathrm{p}<0.0001)$. Asymptomatic children after caustic ingestion have a very low-probability of moderate-severe endoscopic lesions, and the risk of severe damage increases proportionally with $\geq 2$ symptoms. Prospective studies are needed to evaluate the efficacy of intensive clinical observation versus EGD on low-risk children to identify those with clinically relevant esophageal lesions.
\end{abstract}

\footnotetext{
Correspondence: Emanuele Castagno, S.C. Pediatria d'Urgenza, Ospedale Infantile Regina Margherita, A.O.U. Città della Salute e della Scienza di Torino - Piazza Polonia 94, 10126 Torino, Italy

Tel.: +39.011.3135205 - Fax: +39.011 .3135060$

E-mail: ecastagno@cittadellasalute.to.it
}

Key words: caustics; endoscopy; children.

Acknowledgments: We would like to thank the Division of Pediatric Surgery of the Regina Margherita Children's Hospital of Turin, Italy, for the collaboration and the management of caustic ingestion in children accessing our Emergency Department.

Conflict of interest: the authors declare no potential conflict of interests.

Ethics approval and consent to participate: The manuscript does not contain any individual person's data in any form.

Received for publication: 17 April 2020.

Accepted for publication: 31 May 2020.

This work is licensed under a Creative Commons Attribution 4.0 License (by-nc 4.0).

CO Copyright: the Author(s), 2020

Licensee PAGEPress, Italy

Emergency Care Journal 2020; 16:9044

doi:10.4081/ecj.2020.9044

\section{Introduction}

Caustic Ingestion (CI) is relatively common in childhood, but the distinction between suspected and confirmed cases is often difficult, and sometimes children do not show any sign or symptom. ${ }^{1,2}$ The first step is to identify the ingested caustic, its chemical properties, $\mathrm{pH}$, physical form, concentration, ingested quantity and contact time, as all such features influence the severity of eventual gastrointestinal lesions. . $^{3,4}$

The most efficient method to assess mucosal damage after CI is EsophagoGastroDuodenoendoscopy (EGD), ${ }^{5}$ but the relationship between signs and symptoms and the extent of gastroesophageal damage is still unclear, ${ }^{1,6-9}$ and different studies draw different conclusions about EGD indications and timing. $5,9,10$ Indeed, no strong epidemiological and clinical predictors of severe lesions are reported in literature, apart from suicidal attempt, accidental deliberate ingestion, hematemesis and dyspnea; ${ }^{1,11}$ the predictive role of oral lesions is debated. ${ }^{5,6}$ Available guidelines by the European Society for Pediatric Gastroenterology Hepatology and Nutrition (ESPGHAN) and the European Society of Gastrointestinal Endoscopy (ESGE) recommend that "every child that has ingested a corrosive substance should have a thorough follow-up, with endoscopy dictated by symptoms and dependent on the symptoms/signs, the timing should be within 24 hours". ${ }^{11}$ As regards children with suspected CI and symptoms/signs, EGD is suggested to identify digestive tract lesions, but is withheld if the child is asymptomatic. ${ }^{11}$ The Italian Consensus Statement proposed by the Italian multicenter observational study of the Gastroenterology, Hepatology and Nutrition Society (SIGENP) provides similar recommendations. ${ }^{12}$

Aims of this study were to determine the predictive value of epidemiological and clinical features of CI and to evaluate guidelines adherence.

\section{Materials and Methods}

This single-center retrospective observational study was conducted on all the patients $<18$ years referred to the Emergency Department (ED) of the Regina Margherita Children's Hospital of Turin, Italy, since January 2012 to December 2017 because of acid $(\mathrm{pH}<2)$, alkaline $(\mathrm{pH}>12)$, oxidants agents and/or household bleach ingestion. Although household bleach is an alkaline agent, it was considered separately because its toxic action is partly due also to oxidizing effect.

The patients were identified reviewing our Hospital medical records; the following International Classification of Diseases $\left(9^{\text {th }}\right.$ revision) discharge codes were screened: 947 (other and unspecified drugs and medical substances causing adverse effects in therapeutic use), 983.0 through 983.9 (toxic effects of corrosive aromatic acids and caustic alkalis), 989.0 through 989.89 (toxic 
effects of substances chiefly non-medicinal), 530.1 through 530.8 (other specified disorders of the esophagus), 535.4 (other specified gastritis), E861.0 through E861.9 (accidental poisoning by cleansing and polishing agents, disinfectants paints and varnishes), E864.0 through E864.4 (accidental poisoning by corrosives and caustic not elsewhere classified), E866 (accidental poisoning by other metals and their compounds and fumes), E950.7 (suicide and self-inflicted poisoning by corrosive and caustic substances).

Each event was classified as accidental or intentional (suicidal attempts). Accidental events were defined as unintentional selfexposure to caustics (unintentional general) or when unaware children deliberately drank a liquid in cans or bottles containing caustics (accidental-deliberate).

All the children were divided in four groups according to age: $<1$ year, 1-4 years, 5-10 years, $>10$ years. The information recorded for each admission were time of arrival, time between the event and hospital admission, length of stay in the ED, admission to Intensive Care Unit, commercial name and characteristics of the caustic agent(s) involved in each event, packaging (original or non-original), recurrence of poisoning, site of the event, and whether CI was accidental or intentional. For each patient we also recorded clinical, diagnostic and therapeutic data.

The severity of signs and symptoms was graded according to the Poisoning Severity Score (PSS). ${ }^{13}$ Based on PSS, we divided gastrointestinal and respiratory signs and symptoms into mild (one single vomiting, oral and/or pharyngeal lesions), moderate (prolonged vomiting, dysphagia, drooling, hematemesis, dyspnea) and severe (massive hemorrhage with shock and/or perforation, death). Induced vomiting was excluded.

Adherence to ESPGHAN/ESGE guidelines and the Italian Consensus for EGD and therapy was evaluated. ${ }^{11,12}$ EGD is indicated in case of intentional or accidental-deliberate CI with or without symptoms; $\geq 1$ sign(s) or symptom(s) suggestive for possible oropharyngeal, esophageal and gastric lesions; respiratory symptoms. Clinical observation for $24-48$ hours is suggested as first-line approach instead of EGD when CI is only suspected and children are asymptomatic. ${ }^{12}$

EGD timing after CI was classified in urgent ( $<6$ hours) or deferred (6-24 hours or more). Gastroesophageal lesions were classified by endoscopic classification modified from Zargar (Table 1). ${ }^{14}$

As regards therapy, for each patient we recorded the type of gastroprotection (antacids, including magnesium hydroxide/aluminum hydroxide, magaldrate anhydrous, and sucralfate; H2blockers; proton pump inhibitors), and if antibiotic and steroids were given. Therapeutic adherence to guidelines and consensus has been evaluated according to the following recommendations: no therapy for grade I lesions, proton pump inhibitors for grade II, corticosteroids for grade $\mathrm{IIb}^{10}$ and $\mathrm{III}^{11}$ and antibiotics for grade III.

Quantitative data have been described as mean \pm Standard Deviation (SD) or median(range), as appropriate; qualitative data have been described using frequencies and percentages. Differences between groups were tested by Chi-squared test or Fisher's exact test, as appropriate. Correlation was tested by Spearman cograduation coefficient. All data were analyzed using SAS Software for Windows, version 9.2 (SAS Institute Inc., Cary, NC, USA). All the tests were two-tailed and statistical significance was set at $\mathrm{p}<0.05$.

The study protocol conforms to the International regulatory guidelines and current codes of Good Epidemiological Practice. Because patients' care was not altered by the inclusion in the study, the approval of ethical committee was not necessary.

\section{Results}

Out of 271,235 total ED visits during the study period, 107 children $(0.04 \%)$ have been admitted due to acute CI, accounting for $9.93 \%$ of all the acute poisoning. Among them, 63/107 (58.88\%) were males and 79/107 were $1-4$ years old $(73.83 \%)$; median age was 2.17 years (range $0.60-16.08$ years).

\section{Epidemiological features}

Epidemiological features of the study population are reported in Table 2. Overall, 94 CI were accidental $(87.85 \%)$ and 13 were intentional suicide attempts $(12.15 \%)$; all the 79 children ageing 1 4 years experienced accidental CI (84.04\%), while $92.31 \%$ of suicide attempts involved children $>10$ years $(p<0.0001)$. There was significant prevalence of males for accidental cases and females for suicide attempts $(\mathrm{p}=0.037)$.

Information regarding the packaging of the caustic agent was unknown in 53/94 accidental CI (56.38\%). Focusing on children with moderate-severe accidental injuries, the packaging was not known in $5 / 10$ cases $(50.00 \%)$ or did not correspond to the original in the other $5 / 10$ cases $(50.00 \%)$.

All the caustics were in liquid form. The most common agent was household bleach, involved in 50/107 cases (46.73\%); 32/107 were alkaline (29.91\%), 12/107 were acid (11.21\%), and $11 / 107$ oxidant (10.28\%); the agent was unknown in $2 / 107$ cases $(1.87 \%)$. No significant correlation was reported between age, gender, time since ingestion, characteristics of caustics and clinical signs and symptoms.

\section{EGD findings}

EGD was performed on 46/107 children (42.99\%) and was positive in $25 / 46$ cases $(54.30 \%)$. On the other hand, $35 / 61$ children $(57.38 \%)$ who should have undergone EGD, based on ESPGHAN/ESGE guidelines and the Italian Consensus, ${ }^{10,11}$ actually did not. Adherence for EGD, when performed, was complete for each grade of lesions. Out of the 21/46 patients with no lesions, only one had no indication for EGD $(\mathrm{p}<0.0001)$.

Among children who underwent EGD, mild (grade I), moderate (grade II), and severe lesions (grade III) were reported in 10/46 $(21.74 \%), 11 / 46(23.91 \%)$, and $4 / 46(8.70 \%)$ respectively. Two cases of esophageal stricture were described among severe lesions: one in a 2-year-old girl with accidental-deliberate ingestion of household product containing sodium hypochlorite and sodium hydroxide, and one in a 14-year-old girl who had ingested a household cleaning product containing ammonia, as suicide attempt. Gastric burns were reported in $11 / 46$ patients $(23.91 \%) ; 3 / 11$ showed also duodenal abnormalities, but no one was related to acid agents. Eight/15 children with grade II and III lesions (53.33\%) ingested alkaline agents, $6 / 15(40.00 \%)$ ingested household bleach and $1 / 15(6.67 \%)$ an acid agent.

Out of the 50 children who ingested household bleach, 18 (36.00\%) underwent EGD: 12/18 (66.67\%) showed no or grade I lesions, and 6/18 (33.33\%) showed grade II or III lesions. Out of the 32 children who ingested alkaline agents, 21 (65.62\%) underwent EGD: $13 / 21$ (61.90\%) showed no or grade I lesions, and 8/21 (38.10\%) showed grade II or III lesions. Comparing the proportion of grade II/III lesions between children who ingested bleach and alkaline agents, no significant difference was observed $(p=0.76)$. Three/12 children underwent EGD after the ingestion of acid agents: $2 / 3(66.67 \%)$ had no or grade I lesions, and 1/3 (33.33\%) had grade II lesions. Three/11 children underwent EGD after the ingestion of oxidant agents, and all of them had no or grade I lesions (Table 3). 
Among the 21 patients with no lesions, 5 (23.81\%) underwent EGD between 3 and 6 hours after the ingestion, $15(71.43 \%)$ between 6 and 24 hours and $1(4.76 \%)$ over 24 hours. All the 10 patients with grade I lesions and 7/11 patients with grade II lesions (64.63\%) underwent EGD between 6 and 24 hours. All the 4 patients with grade III lesions underwent EGD within 6 hours. One case was the suicide attempt of a 13-year-old girl who ingested 15 $\mathrm{ml}$ of bleach, and showed single vomiting; the other 3 cases were accidental: a 2-year-old boy, who swallowed a degreaser for industrial scrubbers without evidence of sequelae at the chest X-ray and showed vomiting, oral lesions and cough, and two siblings (3-yearold boy and 2-year-old girl) who swallowed a large amount of household product containing sodium hypochlorite and sodium hydroxide; the boy showed oral lesions, cough and single vomiting, and resulted in esophageal perforation, while his younger sister showed single vomiting and presented tight esophageal stenosis, as already described above. No fatal cases were reported.

Endoscopic follow-up was correctly performed in all the patients with grade III lesions; it was performed also in $7 / 11$ patients with grade II lesions (63.64\%) and in $1 / 10$ with grade I lesions $(10 \%)$ even if not strictly indicated $(\mathrm{p}<0.0001)$.

\section{Correlation between clinical features and EGD findings}

Among the children with no lesions or grade I lesions, respectively $14 / 21(66.67 \%)$ and $7 / 10(70.00 \%)$ patients presented almost 1 symptom upon arrival $(\mathrm{p}<0.0002)$. The absence of symptoms was always associated with no or mild lesions $(p<0.0002)$. The PSS value correlated with moderate-severe clinical consequences (PSS 2 or 3 ) for all the patients with grade II and III lesions $(\mathrm{p}<0.0001)$. All the children whose management was modified by grade II and III lesions presented at least 1 symptom at the onset.

Oral and/or pharyngeal lesions correlated with moderatesevere EGD injuries, as they were reported in $72.73 \%$ of grade II and $50 \%$ of grade III lesions $(\mathrm{p}<0.003)$. Moderate gastric symptoms were reported in 4 patients with grade I $(40.00 \%)$, in 9 patients with grade II $(81.82 \%)$, but in no patient with grade III lesions $(\mathrm{p}<0.0001)$.

Among moderate symptoms, drooling and prolonged vomiting episodes were significant predictors of mild and moderate EGD injuries $(p<0.0104$ and $p<0.0089$ respectively) but they were not significant predictors of severe EGD injuries. Prolonged vomiting showed moderate positive linear correlation to the presence of lesions of any grade $(\rho=0.37)$.

The risk of moderate-severe EGD injuries increased progressively with increasing number of signs and symptoms: $\geq 2$ symptoms showed a good positive correlation with lesion severity $(\mathrm{p}<0.0002)$. Among the 9 children who underwent EGD with only 1 symptom, 7 (77.78\%) had ingested alkaline agents; 4/9 (44.44\%) showed grade I lesions (2 reported prolonged vomiting, 1 stom- achache and 1 oral lesions), 3/9 showed grade II lesions (1 with prolonged vomiting, 1 with hematemesis and 1 with dysphagia) and 2/9 children showed grade III lesions (both with prolonged vomiting). No case of dyspnea was reported (Table 4).

\section{Laboratory tests}

Overall, 63/107 children (58.88\%) had blood tests (blood cells count, liver and kidney function, C-reactive protein, electrolytes, lactate and $\mathrm{pH}$ ), but only in 3 cases the results modified the management. The first was the above reported 13-year-old girl who ingested household bleach as suicide attempt; she was put on antibiotic because of high white blood cells count and high C-reactive protein level. The second was a 16-month-old boy who accidentally ingested toilet cleaner with hydrochloric acid; he showed high white blood cells count, high C-reactive protein level and low plasmatic sodium and was put on i.v. antibiotic and hydration. The last one was a 3-year-old boy who accidentally ingested a household alkaline cleaner and showed high white blood cells count and high C-reactive protein level; he was put on antibiotic as well.

\section{Therapy}

As regards therapy, there was complete adherence to the Italian Consensus only for patients with grade III lesions; no patients with grade I and 4/11 (36.36\%) patients with grade II followed the Italian Consensus $(\mathrm{p}<0.0001)$. All the 10 patients with grade I lesions and 18/21 (85.71\%) patients with no lesions received gastric antacid therapy alone or in addition to proton pump inhibitors. Antibiotics were used in 5 patients $(45.45 \%)$ with grade II and in 1 patient who did not undergo EGD. If we considered ESPGHAN/ESGE guidelines, ${ }^{10}$ which recommend steroids for patients with grade IIb lesions, the adherence was complete for $5 / 11$ patients $(45.45 \%)$. The type of medication administered for each grade of injury is shown in Table 5.

\section{Discussion}

Our study shows that the absence of signs and symptoms after $\mathrm{CI}$ is always associated with no or minimal gastroesophageal lesions. On the other side, the association of $\geq 2$ symptoms after CI was positively associated with moderate-severe lesions at EGD. This is consistent both with retrospective and prospective previous studies reporting a very low incidence of patients with grade III lesions without any early symptoms and/or signs. ${ }^{1,2,10,15,16}$ Indeed, more than half of the children with mild-moderate signs or symptoms who should had EGD based on available recommendation, actually did not, but also did not show any worsening during intensive clinical observation and were discharged home. Among them, no one developed any complication on follow-up.

Table 1. Zargar Endoscopic classification of gastroesophageal injury.

\begin{tabular}{ll} 
Degree & Endoscopic findings \\
\hline 0 & Normal examination \\
I & Edema and hyperemia of the mucosa \\
\hline II a & Superficial ulceration, erosions, friability, blisters, exudates, hemorrhages, whitish membranes \\
II b & Grade 2a plus deep discrete or circumferential ulcerations \\
\hline III a & Small scattered areas of multiple ulceration and areas of necrosis with brown-black or greyish discoloration \\
III b & Extensive necrosis \\
\hline
\end{tabular}


It is well known that no sign or symptom is completely predictive of relevant gastroesophageal injury at EGD, ${ }^{12,17}$ and the risk of severe lesions increases proportionally with the number of signs and symptoms reported. ${ }^{1,11}$ Indeed, EGD may prove to be particularly valuable in the event of large amounts of caustic agent ingested, suicide attempts, or persistent symptoms. ${ }^{18}$

Among mild symptoms, oropharyngeal lesions are the only which correlate significantly with higher risk of moderate-severe endoscopic injuries, as already described. ${ }^{1,7,16}$ Moderate symptoms did not correlate with lesions' severity, as drooling and prolonged vomiting were observed also in patients with no lesions. On the other side, prolonged vomiting was reported by the majority of children undergoing EGD because of just one single symptom. Unfortunately, the small number of such children in our population does not allow any statistical evaluation, and we cannot exclude that the high variability of lesions' degrees reported was casual. Hematemesis has a high positive predictive value for gastroesophageal lesions, but in our study, it was reported only in 1 patient with grade II lesions. ${ }^{1,10,11}$

Endoscopy is recommended for all symptomatic children with suspected CI, regardless signs and symptoms severity. ${ }^{11,12}$ On the other hand, some authors have suggested wait-and-see strategy in selected patients in order to avoid unnecessary EGD, and have shown that conservative approach can be safe in case of mild symptoms and ingestion of specific groups of corrosive agents. ${ }^{2,4,8}$ Anyway, the decision whether to perform EGD should be made by ED pediatricians along with trained pediatric gastroenterologist, in order to avoid excessive delay in the treatment of severe caustic lesions. ${ }^{10}$ On our results, we could argue if EGD could be reasonably withheld in favor of intensive clinical observation and followup not only in asymptomatic patients, but also in well-selected population of children with accidental non-deliberate CI and mildmoderate symptoms without oral lesions. Unfortunately, this hypothesis cannot be completely answered by our observation: prospective multicenter studies are needed to evaluate the efficacy of intensive clinical observation versus EGD on low-risk children to identify those with clinically relevant esophageal lesions.

Laboratory tests are recommended especially in critical patients, ${ }^{2,18}$ but are unlikely to influence the treatment of caustic ingestion $^{2}$. Consistently with this observation, in our experience the management was modified by laboratory findings only in three cases.

As regards epidemiological features of pediatric CI, available data are scanty. ${ }^{19,20}$ Information about the real amount of ingested caustic and effective ingestion may be not completely reliable, as caregivers do not witness most of CI. Anyway, usually a small amount of caustic agent is involved in unintentional general $\mathrm{CI}^{2}$, often resulting in oral contamination instead of complete ingestion.

Previous Italian reports show that CI accounts for $11 \%$ of poi- soned children referring to ED, and that household bleach is the most commonly ingested agent, consistently with our observation. ${ }^{15,21}$ Commercial household bleach, containing sodium hypochlorite and sodium hydroxide, is an alkaline agent with oxidizing properties. It is known to cause minor adverse effects when ingested: ${ }^{19,22}$ usually, it causes mild late injuries and rarely penetrate deeply enough to damage the submucosa or muscularis propria. ${ }^{23} \mathrm{~A}$ few cases of fatal bleach ingestion are described. ${ }^{24}$ In our study, household bleach ingestion resulted mostly in none or grade I lesions; only the large amount of chlorine bleach's ingestion by suicidal attempts or accidental-deliberate CI showed severe lesions, and none of them showed persistent vomiting.

As already reported, alkalis were the most responsible for injuries, even in children reporting just one single symptom. Anyway, surprisingly, the proportion of moderate-severe lesions in

Table 2. Baseline characteristics of the patients included in the study.

\begin{tabular}{lc} 
Variable & n (\%) \\
Age (years) & \\
$<1$ & $3(2.80)$ \\
$1-4$ & $79(73.83)$ \\
$5-9$ & $10(9.35)$ \\
$\geq 10$ & $15(14.02)$ \\
Gender & \\
Male & $63(58.88)$ \\
Female & $44(41.12)$ \\
\hline Intention & \\
Accidental & $94(87.85)$ \\
Intentional & $13(12.15)$ \\
Caustic type & \\
Household bleach & $50(46.73)$ \\
Alkaline & $32(29.91)$ \\
Acid & $12(11.21)$ \\
Oxidant & $11(10.28)$ \\
Unknown & $2(1.87)$ \\
\hline Time since ingestion (hours) & \\
6-11 & $19(17.76)$ \\
12-17 & $39(36.45)$ \\
18-23 & $36(33.64)$ \\
0-5 5 Unknown & $2(1.87)$ \\
Packaging (excluding suicidal attempts) & $11(10.28)$ \\
Original & \\
Not original & $17 / 94(18.09)$ \\
Unknown & $24 / 94(25.53)$ \\
\hline
\end{tabular}

Table 3. Correlation between caustic type and grade of gastroesophageal lesions. The different proportion of grade II/III between children who ingested household bleach and alkaline agents was not significant $(p=0.76){ }^{\$}$

\begin{tabular}{|c|c|c|c|c|c|c|}
\hline Caustic type (total 107) & $\begin{array}{c}\text { Number } \\
\text { n }\end{array}$ & $\begin{array}{c}\text { no EGD* } \\
\text { n }(\%) \div\end{array}$ & $\begin{array}{c}\text { Grade } 0 \\
\text { n }(\%) \div\end{array}$ & $\begin{array}{l}\text { Grade I } \\
\text { n (\%) } \div\end{array}$ & $\begin{array}{c}\text { Grade II } \\
\text { n (\%) } \%\end{array}$ & $\begin{array}{c}\text { Grade III } \\
\text { n }(\%) \div\end{array}$ \\
\hline Household bleach ${ }^{\S}$ & 50 & $32(64.00)$ & $8(16.00)$ & $4(8.00)$ & $5(10.00)$ & $1(2.00)$ \\
\hline Alkaline ${ }^{\S}$ & 32 & $11(34.38)$ & $10(31.25)$ & $3(9.37)$ & $5(15.63)$ & $3(9.37)$ \\
\hline Acid & 12 & $9(75.00)$ & $1(8.33)$ & $1(8.33)$ & $1(8.33)$ & $0(0.00)$ \\
\hline Oxidant & 11 & $8(72.73)$ & $2(18.18)$ & $1(9.09)$ & $0(0.00)$ & $0(0.00)$ \\
\hline Unknown & 2 & $1(50.00)$ & $0(0.00)$ & $1(50.00)$ & $0(0.00)$ & $0(0.00)$ \\
\hline
\end{tabular}

*no EGD: patients who did not undergo esophagogastroduodenoendoscopy. $¥$ Proportion of cases for each kind of caustic agent. 
our population was not significantly different between children exposed to household bleach and those exposed to alkaline agents. This observation could be affected by the small size of our population. Moreover, the majority of our cases were due to exploratory accidental ingestions, usually involving small amounts of caustics, as noted above. The potential damage of alkaline agents is often worse because of their innocuous taste, leading to the ingestion of larger amounts; liquefactive necrosis and subsequent submucosal destruction allows deeper penetration, causing more esophageal lesions than acids. ${ }^{2,6,25,26}$ Experimental models on animals have shown that alkaline agents may determine worsening of damage within a couple of hours since mucosal contact, ${ }^{27}$ suggesting that too early EGD could underestimate esophageal lesions. In our population, 4 out of 5 patients with no lesion on EGD had ingested an alkaline agent, but endoscopy was never earlier than 3 hours after ingestion, so we are confident that they are unlikely false negatives.

Finally, among accidental CI, the packaging was unknown in $56.38 \%$ of cases, maybe due to the retrospective nature of this study. Anyway, non-original packaging (e.g. bottle of common use without label) was reported in $25.53 \%$ of cases, and put children on higher risk of accidental ingestion. The storage of caustics in unlabeled non-original packaging still represent a major cause of severe CI and should be stressed in preventing campaigns.
Advising parents to place caustics out of reach, and childproof packaging could be a step forward in the prevention of CI. ${ }^{7}$

The main limits of our study were its single-center, retrospective nature, with low sample size of patients who had EGD, and scanty evidence of effective ingestion instead of oral contamination by a small amount of caustic agent, as we do not know if caregivers actually witnessed the majority of episodes. Moreover, just little information on the packaging of caustic agents was known.

\section{Conclusions}

In conclusion, our study shows that children with no symptoms after CI have a very low-probability of moderate-severe endoscopic lesions, and the risk of severe lesions increases proportionally with the presence of $\geq 2$ signs and/or symptoms, in particular drooling and prolonged vomiting. Diagnostic recommendations were often unattended; complete adherence on therapy was reported only for patients with grade III lesions on EGD, and overtreatment was observed for minor injuries.

Large prospective multicenter studies are needed to evaluate the efficacy of intensive clinical observation versus EGD on lowrisk children to identify those with clinically relevant esophageal lesions.

Table 4. Correlation between single and grouped gastrointestinal signs and symptoms and grade of gastroesophageal lesions.

\begin{tabular}{|c|c|c|c|c|c|c|}
\hline $\begin{array}{l}\mathrm{GI}^{\dagger} \text { signs and/or } \\
\text { symptoms }\end{array}$ & $\begin{array}{c}\text { no EGD } \\
\text { (61/107 children) } \\
\text { n (\%) }\end{array}$ & $\begin{array}{c}\text { Grade } 0 \\
\text { (21/107 children) } \\
\text { n (\%) }\end{array}$ & $\begin{array}{c}\text { Grade I } \\
\text { (10/107 children) } \\
\text { n (\%) }\end{array}$ & $\begin{array}{c}\text { Grade II } \\
\text { (11/107 children) } \\
\text { n (\%) }\end{array}$ & $\begin{array}{c}\text { Grade III } \\
\text { (4/107 children) } \\
\text { n (\%) }\end{array}$ & p-value \\
\hline none & $32(52.46)$ & $7(33.33)$ & $3(30.00)$ & $0(0.00)$ & $0(0.00)$ & 0.0002 \\
\hline 1 & $13(21.31)$ & $12(57.14)$ & $4(40.00)$ & $3(27.27)$ & $2(50.00)$ & \\
\hline$\geq 2$ & $16(26.23)$ & $2(9.52)$ & $3(30.00)$ & $8(72.73)$ & $2(50.00)$ & \\
\hline Minor symptoms & $24(39.34)$ & $8(38.10)$ & $4(40.00)$ & $1(9.09)$ & $4(100.00)$ & 0.0316 \\
\hline Oral and/or oropharyngeal lesions & $11(18.03)$ & $6(28.57)$ & $4(40.00)$ & $8(72.73)$ & $2(50.00)$ & 0.0032 \\
\hline Vomiting & $16(26.23)$ & $4(19.05)$ & $2(20.00)$ & $6(54.55)$ & $4(100.00)$ & 0.0076 \\
\hline Moderate symptoms & $3(4.92)$ & $5(23.81)$ & $4(40.00)$ & $9(81.82)$ & $0(0.00)$ & $<0.0001$ \\
\hline Prolonged vomiting & $1(1.64)$ & $3(14.29)$ & $2(20.00)$ & $2(27.27)$ & $0(0.00)$ & 0.0089 \\
\hline Drooling & $2(3.28)$ & $2(9.52)$ & $2(20.00)$ & $4(36.36)$ & $0(0.00)$ & 0.0104 \\
\hline Dysphagia & $0(0.00)$ & $0(0.00)$ & $0(0.00)$ & $1(9.09)$ & $0(0.00)$ & \\
\hline Hematemesis & $0(0.00)$ & $0(0.00)$ & $0(0.00)$ & $1(9.09)$ & $0(0.00)$ & \\
\hline
\end{tabular}

†GI: gastrointestinal; " ${ }^{\ddagger}$ E EGD: patients who did not undergo esophagogastroduodenoendoscopy.

Table 5. Correlation between therapeutic options and grade of gastroesophageal lesions.

\begin{tabular}{|c|c|c|c|c|c|c|}
\hline Therapy & $\begin{array}{c}\text { no EGD } \\
\text { n }(\%)\end{array}$ & $\begin{array}{c}\text { Grade } 0 \\
\text { n (\%) }\end{array}$ & $\begin{array}{c}\text { Grade I } \\
\text { n (\%) }\end{array}$ & $\begin{array}{c}\text { Grade II } \\
\text { n (\%) }\end{array}$ & $\begin{array}{c}\text { Grade III } \\
\text { n (\%) }\end{array}$ & p-value \\
\hline Gastroprotection ${ }^{\ddagger}$ & $54(88.52)$ & $18(85.71)$ & $10(100)$ & $11(100)$ & $4(100)$ & \\
\hline Antacid and/or H2-blockers & $52(85.25)$ & $13(61.9)$ & $7(70.0)$ & $7(63.64)$ & $1(25.0)$ & 0.0149 \\
\hline Proton-pump inhibitors & $6(9.84)$ & $7(33.33)$ & $7(70.0)$ & $10(90.91)$ & $4(100.0)$ & $<0.0001$ \\
\hline Antibiotics & $1(1.64)$ & $0(0.0)$ & $0(0.0)$ & $5(45.45)$ & $4(100.0)$ & $<0.0001$ \\
\hline Steroids & $0(0.0)$ & $1(4.76)$ & $0(0.0)$ & $1(9.09)$ & $4(100.0)$ & $<0.0001$ \\
\hline
\end{tabular}

${ }^{\dagger}$ no EGD: patients who did not undergo esophagogastroduodenoendoscopy. ${ }^{\ddagger}$ Gastroprotection includes: antacid therapy (magnesium hydroxide/aluminum hydroxide, magaldrate anhydrous, sucralfate), H2-blockers and proton-pump inhibitors. Some children received different medications at the same time. 


\section{References}

1. Betalli P, Falchetti D, Giuliani S, et al. Caustic Ingestion Italian Study Group. Caustic ingestion in children: is endoscopy always indicated? The results of an Italian multicenter observational study. Gastrointest Endosc 2008;68:434-9.

2. Hoffman RS, Burns MM, Gosselin S. Ingestion of Caustic Substances. N Engl J Med 2020;382:1739-48.

3. Betalli P, Rossi A, Bini M, et al. Update on management of caustic and foreign body ingestion in Children. Diagn Ther Endosc 2009;2009:969868.

4. Karagiozoglou-Lampoudi T, Agakidis CH, Chryssostomidou $\mathrm{S}$, et al. Conservative management of caustic substance ingestion in a pediatric department setting, short-term and long-term outcome. Dis Esophagus 2011;24:86-91.

5. Gupta SK, Croffie JM, Fitzgerald JF. Is esophagogastroduodenoscopy necessary in all caustic ingestions? J Pediatr Gastroenterol Nutr 2001;32:50-3.

6. Riffat F, Cheng A. Pediatric caustic ingestion: 50 consecutive cases and a review of the literature. Dis Esophagus 2009;22:89-94

7. Boskovic A, Stankovic I. Predictability of gastroesophageal caustic injury from clinical findings: is endoscopy mandatory in children? Eur J Gastroenterol Hepatol 2014;26:499-503.

8. Bosnali O, Moralioglu S, Celayir A, Pektas OZ. Is rigid endoscopy necessary with childhood corrosive ingestion? A retrospective comparative analysis of 458 cases. Dis Esophagus 2017;30:1-7.

9. Temiz A, Oguzkurt P, Ezer SS, et al. Predictability of outcome of caustic ingestion by esophagogastroduodenoscopy in children. World J Gastroenterol 2012;18:1098-103.

10. Lamireau T, Rebouissoux L, Denis D, et al. Accidental caustic ingestion in children: is endoscopy always mandatory? J Pediatr Gastroenterol Nutr 2001;33:81-4.

11. Thomson M, Tringali A, Dumonceau JM, et al. Paediatric Gastrointestinal Endoscopy: European Society for Pediatric Gastroenterology Hepatology and Nutrition and European Society of Gastrointestinal Endoscopy Guidelines. J Pediatr Gastroenterol Nutr 2017;64:133-53.

12. Betalli P, Rossi A. [Consensus statement sulle urgenze endoscopiche non emorragiche in età pediatrica: Progetto Airone.]. Milan, Italy: AQ Edition; 2008. [Article in Italian].

13. Persson H, Sjöberg G, Haines J, et al. Poisoning Severity
Score: grading of acute poisoning. J Toxicol 1998;36:205-13.

14. Zargar SA. The role of fiberoptic endoscopy in the management of corrosive ingestion and modified endoscopic classification of burns. Gastrointest Endosc 1991;37:165-6.

15. Di Nardo G, Betalli P, Illiceto MP, et al. Caustic ingestion in children: one year experience in three Italian referral centers. J Pediatr Gastroenterol Nutr 2020;71:19-22. doi:10.1097/MPG.0000000000002685.

16. Dogan Y, Erkan T, Cokugras FC, et al. Caustic gastroesophageal lesions in childhood: an analysis of 473 cases. Clin Pediatr (Phila) 2006;45:435-8.

17. Alipour Faz A, Arsan F, Peyvandi M, et al. Epidemiologic Features and Outcomes of Caustic Ingestions; a 10-Year CrossSectional Study. Emerg (Tehran) 2017;5:e56.

18. Manes G, Rossi A, Bini M. [L'ingestione di sostanze caustiche: guida al management del paziente acuto.]. Giorn Ital End Dig 2012;35:23-7. [Article in Italian].

19. Contini S, Scarpignato C. Caustic injury of the upper gastrointestinal tract: a comprehensive review. World J Gastroenterol 2013;19:3918-30

20. Sanchez-Ramirez CA, Larrosa-Haro A, Vasquez-Garibay EM, Macias-Rosales R. Socio-demographic factors associated with caustic substance ingestion in children and adolescents. Int $\mathrm{J}$ Pediatr Otorhinolaryngol 2012;76:253-6.

21. Marchi A, Chiossi M, Renier S, et al. [Peculiar features of childhood poisoning and in the maternal-fetal period.]. Ann Ist Super Sanità 2006;42:318-24. [Article in Italian].

22. Chen TY, Ko SF, Chuang JH, et al. Predictors of esophageal stricture in children with unintentional ingestion of caustic agents. Chang Gung Med J 2003;26:233-9.

23. Ducoudray R, Mariani A, Corte H, et al. The damage pattern to the gastrointestinal tract depends on the nature of the ingested caustic agent. World J Surg 2016;40:1638-44.

24. Arevalo-Silva C, Eliashar R, Wohlgelernter J, et al. Ingestion of caustic substances: a 15-year experience. Laryngoscope 2006;116:1422-6.

25. Kurowski JA, Ka M. Caustic ingestions and foreign bodies ingestions in pediatric patients. Pediatr Clin 2017;64:507-24.

26. Park KS. Evaluation and management of caustic injuries from ingestion of acid or alkaline substances. Clin Endosc 2014;47:301-7.

27. Atug O, Dobrucali A, Orlando RC. Critical pH level of lye $(\mathrm{NaOH})$ for esophageal injury. Dig Dis Sci 2009;54:980-7.3 